\title{
On the Newtonian limit of the Weyl tensor
}

\author{
Jürgen Ehlers • Thomas Buchert
}

Received: 25 June 2009 / Accepted: 30 June 2009 / Published online: 12 July 2009

(C) The Author(s) 2009. This article is published with open access at Springerlink.com

\begin{abstract}
In this note we wish to complement some recent work in the cosmological literature concerning the Weyl conformal curvature tensor and its parts. In particular, we shall give a clear-cut definition of the Newtonian limits of electric and magnetic parts of the Weyl tensor. We also discuss that only a subset of the relativistic equations is needed to obtain a closed system of equations in the Newtonian limit.
\end{abstract}

Keywords General relativity $\cdot$ Weyl tensor $\cdot$ Newtonian limit

\section{Introduction}

In a recent paper Bertschinger and Jain [3] attempted to derive a closed Newtonian system for the evolution of fluid quantities (density, expansion, shear, etc.) by looking at the Newtonian limit of the corresponding equations in general relativity. The idea was to obtain "local" evolution equations in terms of a coupled system of ordinary differential equations (while only the initial data are constructed non-locally) in order to simplify the solution of the problem of gravitational motion and to answer the

\section{J. Ehlers}

Max-Planck-Institut für Gravitationsphysik, Albert-Einstein-Institut,

Schlaatzweg 1, 14473 Potsdam, Germany

\section{T. Buchert}

Theoretische Physik, Ludwigs-Maximilians-Universität, Theresienstr. 37, 80333 Munich, Germany

Present Address:

T. Buchert $(\varangle)$

Centre de Recherche Astrophysique de Lyon, CNRS UMR 5574, Université Lyon 1,

9 Avenue Charles André, 69230 Saint-Genis-Laval, France

e-mail: buchert@obs.univ-lyon1.fr 
question whether the gravitational collapse in general ends in sheet-like (oblate), or filamentary (prolate) objects.

Thereafter, a considerable amount of work has been spent on supporting or disproving this goal $[2,6,18,19,23-26]$, and others. Some of these papers are even concerned with new post-Newtonian theories (Bertschinger and Hamilton [2], see the discussion of that paper by Ellis and Dunsby [18]).

We here wish to complement these works by focusing on two aspects of the problem: firstly, we present a clear-cut derivation of the Newtonian limit of fluid evolution equations in a 4-dimensional "frame theory" developed by one of us $[13,14]$. This theory covers both Einstein's and Newton's theory of gravitationally interacting matter and allows to properly define the Newtonian limit. We shall re-address questions related to the magnetic part of the Weyl tensor.

Secondly, we discuss that only a subset of the general relativistic equations is needed to obtain a closed Newtonian system, and we shall establish the relation to the Newtonian Lagrangian framework formulated first by Buchert and Götz [7], in Newtonian cosmology by Buchert [8], and reviewed by Buchert [9] and Ehlers and Buchert [16].

Throughout this paper greek indices run through $0 \ldots 3$, and latin indices through $1 \ldots 3$, the signature of the metric is $(-,+,+,+)$.

\section{The Newtonian limit of the Weyl tensor and its parts}

In the recent cosmological literature there have been discussions on the role of the Weyl conformal curvature tensor, particularly its "magnetic" part, in the Newtonian limit of general relativity. These considerations suffer from the fact that claims are made about that limit without any reference to, or use of, a definition of that limit. However, a clear-cut (and useful!) definition has been given long ago [5, 13, 14, 21,22].

Here we shall employ a "frame-theory" which covers, in a common 4-dimensional spacetime formalism, Newton's as well as Einstein's theory. In it, a parameter $\lambda=c^{-2}$ serves to distinguish between the two theories, and the limit is taken as $\lambda \rightarrow 0$. In that formalism one uses a temporal metric $t_{\alpha \beta}$ and (inverse) spatial metric $s^{\alpha \beta}$, related by:

$$
t_{\alpha \beta} s^{\beta \gamma}=-\lambda \delta_{\alpha}^{\gamma}
$$

In the GR-case, $\lambda>0$ and $g^{\alpha \beta}=s^{\alpha \beta}, g_{\alpha \beta}=-\lambda^{-1} t_{\alpha \beta}$, whereas in Newton's theory, $\lambda=0$ and $t_{\alpha \beta}=t_{, \alpha} t_{, \beta}$, with $t$ the absolute time. In the limit $\lambda \rightarrow 0$, the Lorentz metrics $g_{\alpha \beta},-\lambda g_{\alpha \beta}$ degenerate, corresponding to the fact that the light cones "open up" and convert into the Newtonian hyperplanes of constant absolute time (for details see: Ehlers [13,14]).

In the notation of the frame-theory, the definition of the Weyl tensor for $\lambda>0$ reads:

$$
C_{\beta \gamma \delta}^{\alpha}=R_{\beta \gamma \delta}^{\alpha}-\delta_{[\gamma}^{\alpha} R_{\delta] \beta}-\lambda^{-1}\left\{t_{\beta[\gamma} R_{\delta] \varepsilon} s^{\varepsilon \alpha}+\frac{1}{3} \delta_{[\gamma}^{\alpha} t_{\delta] \beta} R_{\lambda \mu} s^{\lambda \mu}\right\} .
$$


This expression is meaningless for $\lambda=0$. However, if we use the field equation

$$
R_{\alpha \beta}=8 \pi G\left(t_{\alpha \gamma} t_{\beta \delta}-\frac{1}{2} t_{\alpha \beta} t_{\gamma \delta}\right) T^{\gamma \delta}-\Lambda t_{\alpha \beta}
$$

of the frame-theory, valid for $\lambda \geq 0$, to eliminate $R_{\alpha \beta}$ from Eq. (2), we get:

$$
C_{\beta \gamma \delta}^{\alpha}=R_{\beta \gamma \delta}^{\alpha}-8 \pi G\left\{\delta_{[\gamma}^{\alpha} t_{\delta] \lambda} t_{\beta \mu} T^{\lambda \mu}-t_{\beta[\gamma} t_{\delta] \varepsilon} T^{\varepsilon \alpha}-\frac{2}{3} \delta_{[\gamma}^{\alpha} t_{\delta] \beta} t_{\lambda \mu} T^{\lambda \mu}\right\} .
$$

This formula is meaningful even for $\lambda=0$ (the $\lambda^{-1}$-terms in (2) cancel because of the $\lambda$ in (1)). We therefore define the Weyl tensor in the frame-theory by Eq. (4). This definition is appropriate; for if a sequence of GR-solutions has a Newtonian solution as a limit, then the limit of $C^{\alpha}{ }_{\beta \gamma \delta}$ is indeed given by Eq. (4).

In the Newtonian case, $\lambda=0$, (4) simplifies, because of $t_{\alpha \beta}=t_{, \alpha} t_{, \beta}$, to

$$
C_{\beta \gamma \delta}^{\alpha}=R_{\beta \gamma \delta}^{\alpha}-\frac{8 \pi G}{3} \rho t_{, \beta} \delta_{[\gamma}^{\alpha} t_{, \delta]}
$$

The "electric" and "magnetic" parts of $C^{\alpha}{ }_{\beta \gamma \delta}$ with respect to any 4-velocity $u^{\alpha}$ follow from (5); they read:

$$
\begin{aligned}
& E_{\gamma}^{\alpha}=R_{\beta \gamma \delta}^{\alpha} u^{\beta} u^{\delta}-\frac{4 \pi G}{3} \rho\left(\delta_{\gamma}^{\alpha}-u^{\alpha} t_{, \gamma}\right) ; \\
& H_{\alpha \gamma}=\frac{1}{2} \eta_{\alpha \beta \lambda \mu} s^{\mu \nu} C^{\lambda}{ }_{\nu \gamma \delta} u^{\beta} u^{\delta}=0 .
\end{aligned}
$$

The vanishing of $H_{\alpha \beta}$ in the Newtonian limit can be understood more physically as follows: In GR, $H_{\alpha \beta}$ measures the relative rotation of nearby, freely falling gyroscopes due to gravitomagnetism [27, Appendix ${ }^{1}$ ]. This effect is absent in Newton's theory in which the parallelism of spatial vectors is path independent, in other words, parallel gyroscopes remain parallel if subject to nothing but inertia and gravity.

Investigation of the general case of exact solutions of general relativity with $H_{\alpha \beta}=$ 0 and $\omega_{\alpha \beta}=0$ has been attempted by Barnes and Rowlingson [1]. However, they have not investigated the propagation of the dynamical constraint $H_{a b}=0$, which is needed for statements about classes of exact solutions. ${ }^{2}$ Further investigations of such classes of motion termed "silent universes" may be found in Croudace et al. [11], Bruni et al. [6], Matarrese [25] (and ref. therein), and van Elst and Uggla [31].

\footnotetext{
1 The relevant formula contains a misprint: the correct version is $H_{[\mathrm{g}]}^{a}=\frac{1}{2} \eta^{a i \ell m} R_{\ell m j k} u_{i} \delta x^{j} u^{k}$.

2 This issue was considered later with the additional assumption $p=0 \Rightarrow \dot{u}^{a}=0$ in van Elst et al. [30], where for the general case without any symmetries and Weyl tensor of Petrov type I no complete results could be obtained.
} 


\section{Discussion of the equations in the Newtonian limit}

In 3-dimensional notation, (6) yields, as expected, the Newtonian tidal tensor defined as the trace-free part of the gravitational field tensor $\left(g_{i, j}\right)$ (a comma denotes derivative with respect to Eulerian coordinates):

$$
E_{i j}:=g_{i, j}-\frac{1}{3} \delta_{i j} g_{\ell, \ell}
$$

with

$$
E_{[j i]}=0 ; \quad E_{i i}=0 .
$$

Like any Eulerian field, the Newtonian tidal tensor of the gravitational field strength $\vec{g}$ can be written explicitly in terms of Lagrangian coordinates as follows:

$$
E_{i j}=g_{i \mid k} J_{k j}^{-1}-\frac{1}{3} \delta_{i j} g_{\ell \mid k} J_{k \ell}^{-1}
$$

where a vertical slash denotes derivative with respect to Lagrangian coordinates.

Introducing the diffeomorphic mapping $\vec{f}_{t}: \vec{x}=\vec{f}(\vec{X}, t)$, which sends fluid elements from their (initial) Lagrangian position $\vec{X}$ to a point $\vec{x}$ in Eulerian space at time $t$, and using the expression for the Jacobian of the inverse mapping $\vec{h}=\vec{f}^{-1}$,

$$
\begin{gathered}
\vec{g}[\vec{x}, t]:=\ddot{\vec{f}}(\vec{h}[\vec{x}, t], t), \quad J:=\operatorname{det}\left(f_{i \mid k}\right), \\
h_{j \mid \ell}=\frac{1}{2 J} \epsilon_{\ell p q} \epsilon_{j r s} f_{p \mid r} f_{q \mid s}
\end{gathered}
$$

we can write the tidal tensor explicitly in terms of $\vec{f}$ :

$$
E_{i j}=\frac{1}{2 J}\left(\epsilon_{j p q} \mathcal{J}\left(\ddot{f}_{i}, f_{p}, f_{q}\right)-\frac{1}{3} \epsilon_{o p q} \mathcal{J}\left(\ddot{f}_{o}, f_{p}, f_{q}\right) \delta_{i j}\right)
$$

Thus, any trajectory field $\vec{f}$ which obeys the Lagrange-Newton system (Buchert and Götz [7] $(\Lambda=0)$ and Buchert [8] $(\Lambda \neq 0)$; Ehlers and Buchert [16]),

$$
\begin{gathered}
\mathcal{J}\left(\ddot{f}_{j}, f_{j}, f_{k}\right)=0, \\
\mathcal{J}\left(\ddot{f}_{1}, f_{2}, f_{3}\right)+\mathcal{J}\left(\ddot{f}_{2}, f_{3}, f_{1}\right)+\mathcal{J}\left(\ddot{f}_{3}, f_{1}, f_{2}\right)-\Lambda J=-4 \pi G \stackrel{0}{\rho},
\end{gathered}
$$

determines the evolution of the tidal tensor via (10).

In (14) and (15), $\mathcal{J}(\mathcal{A}, \mathcal{B}, \mathcal{C})$ denotes the functional determinant of any three functions $\mathcal{A}(\vec{X}, t), \mathcal{B}(\vec{X}, t)$ and $\mathcal{C}(\vec{X}, t)$ with respect to Lagrangian coordinates $\vec{X}, \stackrel{0}{\rho}$ is the initial density field and $\Lambda$ the cosmological constant. 
We may use Eq. (13) to state the Lagrange-Newton system (14) and (15) in a different way: it is equivalent to the condition that $E_{i j}$ is symmetric and tracefree [9]: We insert (15) into (13) and write

$$
E_{i j}=\frac{1}{2 J} \epsilon_{j p q} \mathcal{J}\left(\ddot{f}_{i}, f_{p}, f_{q}\right)-\frac{1}{3}\left(\Lambda-\frac{4 \pi G}{J} \stackrel{0}{\rho}\right) \delta_{i j} .
$$

Then,

$$
\begin{aligned}
E_{[i j]} & =0 \Leftrightarrow(14) ; \\
E_{i i} & =0 \Leftrightarrow(15) .
\end{aligned}
$$

This demonstrates that no equation involving $H_{i j}$ is needed in the Newtonian limit to close the system. Indeed, for $H_{\alpha \beta}=0$, the relativistic system of equations for the fluid variables expansion $(\theta)$, vorticity $\left(\omega_{\alpha \beta}\right)$, shear $\left(\sigma_{\alpha \beta}\right)$, "electric" $\left(E_{\alpha \beta}\right)$ and "magnetic" $\left(H_{\alpha \beta}\right)$ parts of the Weyl tensor-derived by one of us (Ehlers [12]) and Trümper [29], and reviewed by Ellis [17]—reduces to the system

$$
\begin{aligned}
\dot{\rho} & =-\theta \rho, \\
\dot{\theta} & =\Lambda-\frac{1}{3} \theta^{2}+2\left(\omega^{2}-\sigma^{2}\right)-4 \pi G \rho, \\
\dot{\omega}^{\alpha} & =-\frac{2}{3} \theta \omega^{\alpha}+\sigma^{\alpha}{ }_{\beta} \omega^{\beta}, \\
\dot{\sigma}_{\alpha \beta} & =-\sigma_{\alpha \gamma} \sigma^{\gamma}{ }_{\beta}-\omega_{\alpha} \omega_{\beta}+\frac{1}{3} h_{\alpha \beta}\left(2 \sigma^{2}-\omega^{2}\right)-\frac{2}{3} \theta \sigma_{\alpha \beta}-E_{\alpha \beta}, \\
\dot{E}_{\alpha \beta} & \left.\left.=-h_{\alpha \beta} \sigma^{\gamma \delta} E_{\gamma \delta}-\theta E_{\alpha \beta}+E_{\gamma(\alpha} \omega_{\beta}\right)^{\gamma}+3 E_{\gamma(\alpha} \sigma_{\beta}\right)^{\gamma}-4 \pi G \rho \sigma_{\alpha \beta},
\end{aligned}
$$

where $h^{\alpha \beta}=g^{\alpha \beta}+u^{\alpha} u^{\beta}$ is the spatial projection tensor. Recall that a solution of this closed set of equations delivers at best an approximation that needs to be controlled. Resulting solutions will have to be subjected to the propagation constraint $\dot{H}_{\alpha \beta}=0$ to find the exact solution classes. This constraint will likely leave only highly symmetric solutions like the Lemaître-Tolman-Bondi and the Szekeres models, since, in general, a non-vanishing magnetic part of the Weyl tensor is generated by evolving a system with initially vanishing magnetic part.

In the Newtonian limit $\lambda \rightarrow 0$ the spatial parts of the Eqs. (19)-(22) are equivalent to those derived from Newtonian theory (compare Szekeres and Rankin [28] and the discussions by Kofman and Pogosyan [19], Lesame et al. [23], Buchert [9] and Matarrese [25]). The Lagrange-Newton-System (14) and (15) can be already obtained from (19)-(21) together with the decomposition $\sigma_{i j}+\omega_{i j}+\frac{1}{3} \theta \delta_{i j}=v_{i, j}$.

Note added 2009 Some examples of Newtonian limits of relativistic spacetimes have been published by Ehlers 1997 together with more details on the Newtonian limit in the frame-theory formalism used here [15]. Furthermore, it is possible to write the Einstein equations in terms of symmetry conditions imposed on the electric and magnetic parts of the Weyl tensor similar to writing the Lagrange-Newton system 
$(14,15)$, since both parts of the Weyl tensor contain all information. The investigation of this formulation together with the corresponding Newtonian limit will be published elsewhere. Related remarks on the Newtonian limit in the framework of Cartan's formulation of Einstein's equations may be found in Buchert [10, Sect. 4.2.1].

Acknowledgments In 1996, TB was supported by the "Sonderforschungsbereich 375-95 für Astro-Teilchenphysik der Deutschen Forschungsgemeinschaft". He would like to thank the Albert-Einstein-Institut in Potsdam for generous hospitality during a working visit in 1996, where this work has been written. TB would also like to thank Henk van Elst for digging out the preprint, as well as for help and comments during the preparation of this manuscript.

Open Access This article is distributed under the terms of the Creative Commons Attribution Noncommercial License which permits any noncommercial use, distribution, and reproduction in any medium, provided the original author(s) and source are credited.

\section{References}

1. Barnes, A., Rowlingson, R.R.: Class. Quantum Gravit. 6, 949 (1989)

2. Bertschinger, E., Hamilton, A.J.S.: Astrophys. J. 435, 1 (1994)

3. Bertschinger, E., Jain, B.: Astrophys. J. 431, 486 (1994)

4. Brauer, U.: J. Math. Phys. 33, 1224 (1992)

5. Brauer, U., Rendall, A., Reula, O.: Class. Quantum Gravit. 11, 2283 (1994)

6. Bruni, M., Matarrese, S., Pantano, O.: Astrophys. J. 445, 958 (1995)

7. Buchert, T., Götz, G.: J. Math. Phys. 28, 2714 (1987)

8. Buchert, T.: Astron. Astrophys. 223, 9 (1989)

9. Buchert, T.: In: Bonometto, S., Primack, J., Provenzale, A. (eds.) Proc. IOP 'Enrico Fermi', Course CXXXII (Dark Matter in the Universe), Varenna 1995, pp. 543-564. IOS Press, Amsterdam (1996)

10. Buchert, T.: Gen. Relativ. Gravit. 40, 467 (2008)

11. Croudace, K., Parry, J., Salopek, D., Stewart, J.: Astrophys. J. 423, 22 (1994)

12. Ehlers, J.: Akad. Wiss. Lit. Mainz, Abh. Math.-Nat. Klasse 11, p. 793 (in German) (1961); translated: Gen. Relativ. Gravit. 25, 1225 (1993)

13. Ehlers, J.: In: Nitsch, J., et al. (eds.) Grundlagenprobleme der Modernen Physik. Bibliograph. Inst. Mannheim, pp. 65-84 (in German) (1981)

14. Ehlers, J.: In: Fenarese, G. (ed.) Classical Mechanics and Relativity: Relationship and Consistency, pp. 95-106. Bibliopolis, Naples (1991)

15. Ehlers, J.: Class. Quantum Gravit. 14, A119 (1997)

16. Ehlers, J., Buchert, T.: Gen. Relativ. Gravit. 29, 733 (1997)

17. Ellis, G.F.R.: In: Sachs, R. (ed.) General Relativity and Cosmology. Academic Press, New York (1971)

18. Ellis, G.F.R., Dunsby, P.K.S.: Astrophys. J. 479, 97 (1997)

19. Kofman, L., Pogosyan, D.: Astrophys. J. 442, 30 (1995)

20. Komar, A.: Phys. Rev. 104, 544 (1956)

21. Künzle, H.P.: Gen. Relativ. Gravit. 7, 445 (1976)

22. Künzle, H.P., Nester, J.M.: J. Math. Phys. 25, 1009 (1984)

23. Lesame, W.M., Ellis, G.F.R., Dunsby, P.K.S.: Phys. Rev. D 53, 738 (1996)

24. Matarrese, S., Pantano, O., Saez, D.: Mon. Not. R. Astron. Soc. 271, 513 (1994)

25. Matarrese, S.: In: Bonometto, S., Primack, J., Provenzale, A. (eds.) Proc. IOP 'Enrico Fermi', Course CXXXII (Dark Matter in the Universe), Varenna 1995, p. 601. IOS Press, Amsterdam (1996)

26. Matarrese, S., Terranova, D.: Mon. Not. R. Astron. Soc. 283, 400 (1996)

27. Sachs, R.K.: Zeitschrift für Physik 157, 462 (1960) (in German)

28. Szekeres, P., Rankin, J.R.: J. Austral. Math. Soc. 20, 114 (1977)

29. Trümper, M.: J. Math. Phys. 6, 584 (1965)

30. van Elst, H., Uggla, C., Lesame, W.M. Ellis G.F.R., Maartens, R.: Class. Quantum Gravit. 14, 1151 (1997)

31. van Elst, H., Uggla, C.: Class. Quantum Gravit. 14, 2673 (1997) 